\title{
Design of magnetic materials: The electronic structure of the ordered, doped Heusler compound $\mathrm{Co}_{2} \mathrm{Cr}_{1-x} \mathrm{Fe}_{x} \mathrm{Al}$
}

\author{
Gerhard H. Fecher ${ }^{1}$, Hem Chandra Kandpal ${ }^{1}$, Sabine \\ Wurmehl $^{1}$, Jonder Morais ${ }^{2}$, Hong-Ji Lin ${ }^{3}$, Hans-Joachim \\ Elmers $^{4}$, Gerd Schönhense ${ }^{4}$, and Claudia Felser ${ }^{1}$ \\ ${ }^{1}$ Institut für Anorganische Chemie und Analytische Chemie, \\ Johannes Gutenberg - Universität, D-55099 Mainz, Germany \\ ${ }^{2}$ Instituto de Fisica, \\ Universidade Federal do Rio Grande do Sul, Porto Allegre, 91501-970 RS, Brazil \\ 3 National Synchrotron Radiation Research Center - NSRRC, \\ Hsinchu, 30076, Taiwan \\ ${ }^{4}$ Institut für Physik, \\ Johannes Gutenberg - Universität, D-55099 Mainz, Germany \\ E-mail: felser@uni-mainz.de
}

\begin{abstract}
Doped Heusler compounds $\mathrm{Co}_{2} \mathrm{Cr}_{1-x} \mathrm{Fe}_{x} \mathrm{Al}$ with varying $\mathrm{Cr}$ to Fe ratio $x$ were investigated experimentally and theoretically. The electronic structure of the ordered, doped Heusler compound $\mathrm{Co}_{2} \mathrm{Cr}_{1-x} \mathrm{Fe}_{x} \mathrm{Al}(x=n / 4, n=$ $0,1,2,3,4)$ was calculated using different types of band structure calculations. The ordered compounds turned out to be ferromagnetic with small $\mathrm{Al}$ magnetic moment being aligned anti-parallel to the $3 d$ transition metal moments. All compounds show a gap around the Fermi-energy in the minority bands. The pure compounds exhibit an indirect minority gap, whereas the ordered, doped compounds exhibit a direct gap. Magnetic circular dichroism (MCD) in X-ray absorption spectra was measured at the $L_{2,3}$ edges of $\mathrm{Co}, \mathrm{Fe}$, and $\mathrm{Cr}$ of the pure compounds and the $x=0.4$ alloy in order to determine element specific magnetic moments. Calculations and measurements show an increase of the magnetic moments with increasing iron content. The experimentally observed reduction of the magnetic moment of $\mathrm{Cr}$ can be explained by $\mathrm{Co}-\mathrm{Cr}$ site-disorder. The presence of the gap in the minority bands of $\mathrm{Co}_{2} \mathrm{CrAl}$ can be attributed to the occurrence of pure $\mathrm{Co}_{2}$ and mixed $\mathrm{CrAl}$ (001)-planes in the $L 2_{1}$ structure. It is retained in structures with different order of the $\mathrm{CrAl}$ planes but vanishes in the $X$-structure with alternating $\mathrm{CoCr}$ and $\mathrm{CoAl}$ planes.
\end{abstract}

PACS numbers: 75.50.Cc, 71.20.Lp, 78.70.Dm, 75.30.Cr

Submitted to: J. Phys.: Condens. Matter 
Figure 1. Structure of ordered $\mathrm{Co}_{2} \mathrm{Cr}_{1-x} \mathrm{Fe}_{x} \mathrm{Al}$ :

a) $x=0,1\left(L 2_{1}\right)$, b) $x=1 / 4(3 / 4$ if exchanging Fe and Cr), and c) $x=1 / 2$ (see text).

\section{Introduction}

A great scientific interest is attracted by materials with a complete spin polarization [1]. Such materials, being a metal for spin up and a semiconductor (or insulator) for spin down electrons, are called half-metallic ferromagnets [2,3] (HMF). Heusler compounds have been considered potential candidates to show this property [2]. Theoretical calculations predicted an energy gap for minority electrons for the halfHeusler compound NiMnSb $[2,4]$ which, however, has been controversially discussed [5-7]. Similarly, a HMF like behaviour was found by Plogmann et al [8] for the Cobalt based Heusler alloy $\mathrm{Co}_{2} \mathrm{MnSn}$.

Heusler compounds belong to a group of ternary intermetallics with the stoichiometric composition $\mathrm{X}_{2} \mathrm{YZ}$ ordered in an $L 2_{1}$-type structure, many of which are ferromagnetic [9]. $\mathrm{X}$ and $\mathrm{Y}$ are transition metals and $\mathrm{Z}$ is usually a main group element. Y may also be replaced by a rare earth element. The cubic structure consists of four interpenetrating fcc lattices. The two fcc sub-lattices of the $\mathrm{X}$ atoms combine to a simple cubic sub-lattice. Remarkably, the prototype $\mathrm{Cu}_{2} \mathrm{MnAl}$ is a ferromagnet even though none of its constituents is one [10]. The $L 2_{1}$ structure of the Heusler compounds is shown in figure 1a.

Ferromagnetic properties of various Heusler compounds have been investigated experimentally $[9,11-15]$ and theoretically $[8,16]$ (see Refs. $[17,18]$ for comprehensive review). The Co-based Heusler compounds $\mathrm{Co}_{2} \mathrm{YZ}$ are of particular interest because they show a comparatively high Curie temperature and varying magnetic moments ranging from $0.3 \mu_{B}$ to $1.0 \mu_{B}$ at the Co site depending on the constituents $\mathrm{Y}$ and $\mathrm{Z}$ (see Refs.: [13,19-21]).

The large variety of possible compositions of the Heusler compounds allows easily to produce materials with predictable magnetic properties. The easiest way to compose new materials is the exchange of one or more of the elements $\mathrm{X}$, Y, or Z. This is indeed widely used in experiments and theory. See Refs. [16, 22-28] for examples on $\mathrm{Co}_{2}$ containing Heusler compounds. However, the differences between the materials are rather rough. A better fine-tuning of magnetic properties may be obtained if substituting one or another constituent only partially.

Such a way to design new materials is possible using a deliberate substitution of elements. Most oftenly the main-group element is kept fixed because the magnetic properties are mainly governed by the transition metal constituents. This leads to alloys of the type $\mathrm{X}_{2-x} \mathrm{Y}_{1+x} \mathrm{Z},\left(\mathrm{X}_{1-x} \mathrm{X}_{x}^{\prime}\right)_{2} \mathrm{YZ}$ or $\mathrm{X}_{2}\left(\mathrm{Y}_{1-x} \mathrm{Y}_{x}^{\prime}\right) \mathrm{Z}(x=0, . ., 1)$. The first type is still a ternary alloy whereas the second and third type result in quaternary alloys. Co based alloys of the first two types have been investigated by various groups $[12,19,29-33]$.

This work focuses on quaternary alloys of the third type. The Cobalt-Aluminium based alloy of the $\mathrm{X}_{2}\left(\mathrm{Y}_{1-x} \mathrm{Y}_{x}\right) \mathrm{Z}$ type with $\mathrm{Y}=\mathrm{Cr}$ and $\mathrm{Y}^{\prime}=\mathrm{Fe}$ is particularly of interest as base for materials design. This arises from the fact that the pure $(x=0$ or 1$)$ compounds exhibit the same lattice parameter within $0.1 \%$. Therefore, substituting partially one element by the other will lead to a material with the same lattice parameter but changed electronic and magnetic properties. Starting from the pure 
Cr containing compound, the partial substitution by Fe may be seen as d-electron doping.

$\mathrm{Co}_{2} \mathrm{Cr}_{0.6} \mathrm{Fe}_{0.4} \mathrm{Al}$ is of special interest because a relatively high magneto-resistance ratio of up to $30 \%$ was found in powder samples in a small magnetic field of $0.1 T$ $[34,35]$. Thin films of the compound were successfully grown by several groups [36-39]. A magneto-resistance ratio of 26.5\% [40] (at 5K) and 19\% [41] (at room temperature) was found for a tunnelling magneto-resistance (TMR) element of the same compound. Very recently, Marukame et al [42] reported a TMR ratio of $74 \%$ at $55 \mathrm{~K}$ for a $\mathrm{Co}_{2} \mathrm{Cr}_{0.6} \mathrm{Fe}_{0.4} \mathrm{Al}-\mathrm{MgO}-\mathrm{CoFe}$ magnetic tunnel junction. A spin polarization of only less than $49 \%$ was found for poly-crystalline samples by means of Andreev reflections [43]. The observation of an incomplete spin polarization may not only be caused by the model used to interpret the data $[43,44]$ but also by the properties of the sample. Clifford et al [45] reported recently a spin polarisation of $81 \%$ in point contacts of $\mathrm{Co}_{2} \mathrm{Cr}_{0.6} \mathrm{Fe}_{0.4} \mathrm{Al}$.

For the purpose of the present study, doped Heusler alloys $\mathrm{Co}_{2} \mathrm{Cr}_{1-x} \mathrm{Fe}_{x} \mathrm{Al}$ were prepared by arc-melting under an argon atmosphere. The resulting specimens were dense poly-crystalline ingots. Structural properties were measured using X-ray diffraction as a standard method. The cubic structure with a lattice constant of about $5.73 \AA$ Âas confirmed for all samples. Flat discs ( $8 \mathrm{~mm}$ diameter by $1 \mathrm{~mm}$ thickness) were cut from the ingots. The discs were mechanically polished for spectroscopic experiments.

Field dependent magnetic properties were measured by SQUID - magnetometry (temperature: $4 K$ to $300 K$ ) and by the magneto-optical Kerr effect (MOKE) at room temperature. The remanent magnetization was less than $10 \%$ of the saturation magnetization pointing on a soft magnetic material. Saturation was achieved for external fields above $0.2 \mathrm{~T}$ at $300 \mathrm{~K}$. Especially the $\mathrm{Co}_{2} \mathrm{CrAl}$ samples showed large differences in the total moment varying from $1 \mu_{B}$ to $3 \mu_{B}$ per formula unit. This depends mostly on the post-processing of the samples like annealing followed either by quenching or slow cooling at different rates. In some cases, X-ray diffraction exhibited pronounced super-structures pointing on a tetragonal distortion of the unit cell. It should be noted that some types of disorder cannot be detected easily by X-ray powder diffraction as the scattering coefficients of $\mathrm{Co}$ and $\mathrm{Cr}$ are very similar. The same applies for neutron diffraction. Due to the nearly equal scattering length of $\mathrm{Cr}$ and $\mathrm{Al}$, in particular, it is not possible to distinguish between ordered $L 2_{1}$ and disordered $B_{2}$ structures. Therefore, we will use a detailed analysis of the magnetic properties to gain information about structural disorder of the samples. It should be noted that a mixing of $\mathrm{Cr}$ and Fe atoms in doped compounds will be hardly detectable by X-ray diffraction. This is caused by the very similar scattering coefficients of the constituting elements. $\mathrm{X}$-ray magnetic circular dichroism (MCD) in soft X-ray absorption spectroscopy was performed at the First Dragon beamline of NSRRC (Hsinchu, Taiwan). The MCD measurements at the $\mathrm{Cr}$, Fe and $\mathrm{Co} L_{2,3}$ absorption edges for $\mathrm{Co}_{2} \mathrm{Cr}_{1-x} \mathrm{Fe}_{x} \mathrm{Al}(x=0$, 0.4 , and 1 ) were carried out in order to investigate element specific magnetic properties and compare them with theoretical predictions. $\mathrm{A} \mathrm{Co}_{2} \mathrm{CrAl}$ sample free of superstructure but with too low magnetic moment was selected for the MCD measurements in order to explain the large deviation from the expected value of $3 \mu_{B}$ per formula unit. $\mathrm{Co}_{2} \mathrm{Cr}_{0.6} \mathrm{Fe}_{0.4} \mathrm{Al}$ was selected for the $\mathrm{MCD}$ measurements as this composition has shown the largest effect in measurements of the magneto resistance.

More details about the experiment and the data analysis are reported in Ref. $[35,46]$. 
The present work reports on calculations of the electronic and magnetic properties of ordered Heusler compounds of the type $\mathrm{X}_{2}\left(\mathrm{Y}_{(1-i / 4)} \mathrm{Y}^{\prime}{ }_{i / 4}\right) \mathrm{Z}$. The calculated properties are compared to experimental values. Deviations from the $L 2_{1}$ structure are discussed on hand of ordered structures. Random alloys of the $\mathrm{X}_{2}\left(\mathrm{Y}_{(1-x)} \mathrm{Y}_{x}^{\prime}\right) \mathrm{Z}$ type with non-rational values of $x$ as well as random disorder (for examples see references $[47,48]$ ) will not be discussed here.

\section{Calculational Details}

Self-consistent band structure calculations were carried out using the scalar relativistic full potential linearised augmented plane wave method (FLAPW) provided by Blaha et al $[49,50]$ (Wien2k). The exchange-correlation functional was taken within the generalized gradient approximation (GGA) in the parametrization of Perdew et al [51]. For comparison, calculations were also performed using the linear muffintin orbital (LMTO) method provided by Savrasov [52] (LMTART 6.5) on different levels of sophistication from simple atomic sphere approximation (LMTO-ASA) to full potential plane wave representation (FP-LMTO-PLW). A $20 \times 20 \times 20 \mathrm{k}$-point mesh was used for the integration in cubic systems.

The properties of the pure $\mathrm{Cr}$ or $\mathrm{Fe}$ containing compounds where calculated in $F m \overline{3} m$ symmetry using the experimental lattice parameter $\left(a=10.822 a_{0 B}\right.$, $a_{0 B}=0.529177 \AA$ ) as determined by X-ray powder diffraction. All muffin tin radii where set to nearly touching spheres with $r_{M T}=2.343 a_{0 B}$ in both full potential methods. The overlapping spheres where set to $r_{M T}=2.664 a_{0 B}$ for the ASA calculations.

The full formula sum of the cubic cell is $\mathrm{X}_{8} \mathrm{Y}_{4} \mathrm{Z}_{4}$, with $\mathrm{X}=\mathrm{Co}, \mathrm{Y}=\mathrm{Cr}$ or $\mathrm{Fe}$ and $\mathrm{Z}=\mathrm{Al}$ and is reduced to $\mathrm{X}_{2} \mathrm{YZ}=\mathrm{Co}_{2} \mathrm{YAl}$. Exchanging $\mathrm{Y}$ and $\mathrm{Z}$ leads indeed to identical structures. (See figure 1 and table 1 for the positions of the atoms.)

The calculation of mixed random alloys is not straight forward in both (FLAPW and LMTO) calculational methods. However, substituting some $\mathrm{Cr}$ atoms of the $L 2_{1}$ structure by Fe leads in certain cases to ordered structures that can be easily used for calculations. Ordered, mixed compounds may have the general formula sum $\mathrm{X}_{8}\left(\mathrm{Y}_{(1-x)} \mathrm{Y}_{x}^{\prime}\right)_{4} \mathrm{Z}_{4}$ with $\mathrm{Y}=\mathrm{Cr}$ and $\mathrm{Y}^{\prime}=\mathrm{Fe}$. These structures have integer occupation of $\mathrm{Y}$ and $\mathrm{Y}^{\prime}$ if $x=i / 4$ with $i=1,2,3$.

Start with the $\mathrm{Cr}$ atoms occupying the corners of the cube and the centre of the faces. The $\mathrm{Al}$ atoms are located at the middle of the cube-edges (see: figure 1a). Replacing the $\mathrm{Cr}$ atom at $(0,0,0)$ by Fe leads to the structure with $x=1 / 4$ (see: figure 1b). The same structure may also be found if starting with $\mathrm{Cr}$ and $\mathrm{Al}$ exchanged and then replacing the $\mathrm{Cr}$ atom at $(1 / 2,1 / 2,1 / 2)$ by Fe. The symmetry of these structures is again cubic but reduced to $P m \overline{3} m$. The only difference is that the base atoms are shifted.

Exchanging simply the $\mathrm{Cr}$ atoms of this structure by Fe leads to the structure with $x=3 / 4$. The accompanied formula sums are $\mathrm{Co}_{8} \mathrm{Cr}_{3} \mathrm{FeAl}_{4}$ and $\mathrm{Co}_{8} \mathrm{CrFe}_{3} \mathrm{Al}_{4}$.

Again, start with the $\mathrm{Cr}$ atoms occupying the corners and face-centres of the cube. Replace two of the face $\mathrm{Cr}$ atom, say at $(0,1 / 2,1 / 2),(1 / 2,0,1 / 2)$ and at opposite faces by Fe. The result is the structure with $x=1 / 2$. This structure is initially cubic but can be reduced to tetragonal symmetry $(P 4 / \mathrm{mmm})$ with the formula sum $\mathrm{Co}_{4} \mathrm{CrFeAl}_{2}$.

The three different structures are illustrated in figure 1. The cell shown for $x=1 / 2$ has a reduced, tetragonal symmetry. The z-axis may be chosen such that the 
Table 1. Symmetry.

Space groups, and Wyckoff positions of the constituents in ordered

$\mathrm{Co}_{2} \mathrm{Cr}_{1-x} \mathrm{Fe}_{x} \mathrm{Al}$.

\begin{tabular}{llcccc} 
compound & & $\mathrm{Co}$ & $\mathrm{Cr}$ & $\mathrm{Fe}$ & $\mathrm{Al}$ \\
\hline $\mathrm{Co}_{2} \mathrm{CrAl}$ & $F m \overline{3} m$ & $8 \mathrm{c}$ & $4 \mathrm{a}$ & - & $4 \mathrm{~b}$ \\
$\mathrm{Co}_{8} \mathrm{Cr}_{3} \mathrm{FeAl}_{4}$ & $P m \overline{3} m$ & $8 \mathrm{~g}$ & $1 \mathrm{a}$ & $3 \mathrm{c}$ & $1 \mathrm{~b}, 3 \mathrm{~d}$ \\
$\mathrm{Co}_{4} \mathrm{CrFeAl}_{2}$ & $P 4 / m m m$ & $4 \mathrm{i}$ & $1 \mathrm{a}$ & $1 \mathrm{~d}$ & $1 \mathrm{~b}, 1 \mathrm{c}$ \\
$\mathrm{Co}_{8} \mathrm{CrFe}_{3} \mathrm{Al}_{4}$ & $P m \overline{3} m$ & $8 \mathrm{~g}$ & $3 \mathrm{c}$ & $1 \mathrm{a}$ & $1 \mathrm{~b}, 3 \mathrm{~d}$ \\
$\mathrm{Co}_{2} \mathrm{FeAl}$ & $F m \overline{3} m$ & $8 \mathrm{c}$ & - & $4 \mathrm{a}$ & $4 \mathrm{~b}$
\end{tabular}

long c-axis coincides with one of the cubic axes of the initial structure. The symmetry and the lattice sites of the structures are summarized in table 1.

Other ordered structures are found from larger elementary cells. The cubic cell doubled in all three directions has the overall formula sum $\mathrm{X}_{64}\left(\mathrm{Y}_{(1-x)} \mathrm{Y}^{\prime}{ }_{x}\right)_{32} \mathrm{Z}_{32}$. The special case with $x=13 / 32$ is very close to the compound $\mathrm{Co}_{2} \mathrm{Cr}_{0.6} \mathrm{Fe}_{0.4} \mathrm{Al}$.

\section{Results and Discussion}

The electronic structure of the pure and doped compounds will be discussed in the following. First, the band structure and the density of states of the ordered compounds are presented. This is followed by a more specific discussion of the magnetic properties on hand of measured and calculated magnetic moments.

A structural optimization was performed for $\mathrm{Co}_{2} \mathrm{CrAl}$ and $\mathrm{Co}_{2} \mathrm{FeAl}$ using FLAPW in order to verify using experimental lattice parameter. The energy minima were found to appear at lattice parameter being less than $0.5 \%$ smaller compared to the experimental values, in both materials. The calculated bulk moduli were $217 \mathrm{GPa}$ and $210 \mathrm{GPa}$ for the $\mathrm{Cr}$ and the Fe containing compounds, respectively. None of the results discussed below changes significantly if using the optimized lattice parameter instead of the experimental one. In particular, the overall spin moments stay the same and the half-metallic behavior of the ordered compounds retains. Very small deviations appear for elemental resolved values. Those are already sensitive to the setting of the $r_{M T}$ and the number of k-points used for integration, as is well known. The only small differences in the observed and optimized structures do not allow to notice any deviation from Vegards law for the mixed compounds.

\subsection{Band structure and density of states}

The self-consistent FLAPW band structure of $\mathrm{Co}_{2} \mathrm{CrAl}$ is shown in figure 2. The energy scale is referenced to the Fermi-energy $\left(\epsilon_{F}\right)$. The typical Heusler gap is located at about $6 \mathrm{eV}$ binding energy. It separates the low lying $\mathrm{s}$ bands from bands of predominately $\mathrm{d}$ character. These low lying s bands emerge mainly from the main group element, here $\mathrm{Al}$. This gap is very small in the $\mathrm{Al}$ containing compounds. Much larger gaps are found for example in Sn containing compounds like $\mathrm{Co}_{2} \mathrm{TiSn}$ [53] or the half-Heusler NiMnSb [54].

From the spin resolved bands, it is seen that the majority bands cross or touch the Fermi-energy $\left(\epsilon_{F}\right)$ in rather all directions of high symmetry. On the other hand, the minority bands exhibit a gap around $\epsilon_{F}$ thus confirming a HMF character. For 


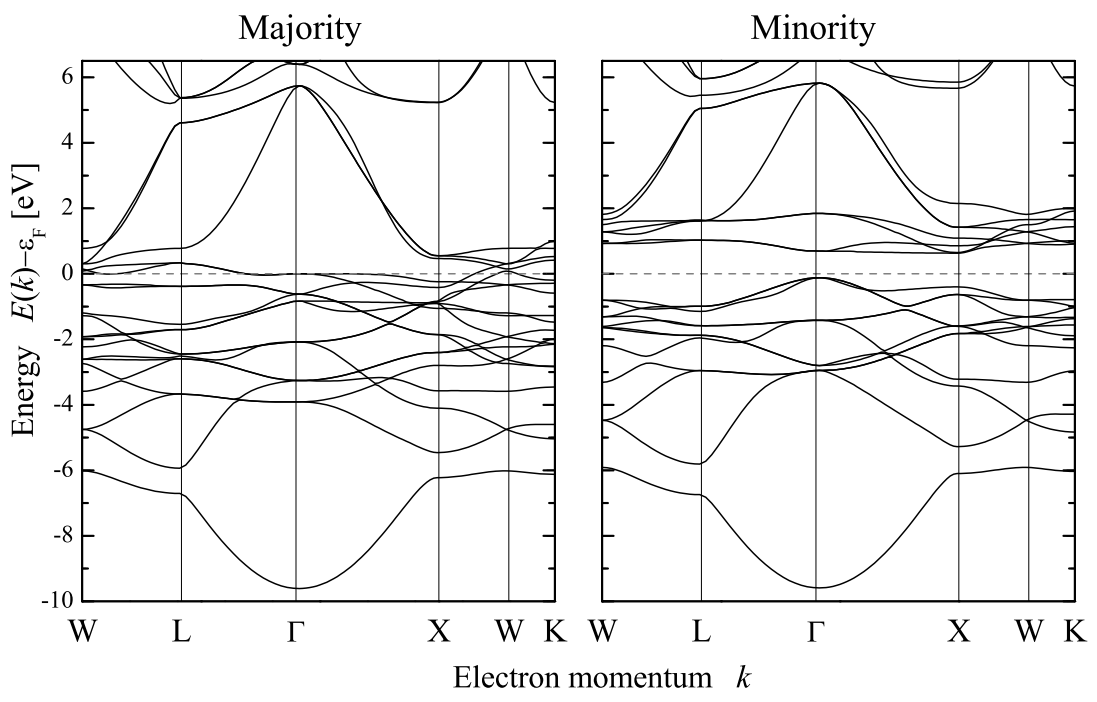

Figure 2. Band structure of $\mathrm{Co}_{2} \mathrm{CrAl}$.

$\mathrm{Co}_{2} \mathrm{CrAl}$, the width of the gap is given by the energies of the highest occupied band at the $\Gamma$-point and the lowest unoccupied band at the $\Gamma$ or $X$-point. The smaller value is found between $\Gamma$ and $X$, thus it is an indirect gap. It should be noted that the direct gap at the $\Gamma$-point is only $60 \mathrm{meV}$ wider. Therefore, a small change in the parameters of the calculation may already change the character of the gap from indirect to direct. Indeed, some LMTO calculations resulted in a direct gap, most probably just for that reason.

We will restrict the following comparison of the doped compounds to the $\Delta$ direction being parallel to [001]. The $\Delta$-direction possesses in all cases $C_{4 v}$ symmetry. It has the advantage that the compound with $x=1 / 2$ can be compared directly to the others even so it is calculated for tetragonal symmetry where the corresponding $\Lambda$-direction is between $\Gamma$ and $Z$.

The $\Delta$-direction is perpendicular to the $\mathrm{Co}_{2}$ (100)-planes. As will turn out later, just the $\Delta$-direction plays the important role for the understanding of the HMF character and magnetic properties of Heusler compounds. This role of the $\Delta$-direction was also pointed out by Öĝüt and Rabe [55].

The band structures in $\Delta$-direction of the pure $(x=0,1)$ and the doped $(x=1 / 2)$ compounds are displayed in figure 3 for energies above the Heusler gap. In general, the doped compounds exhibit much more bands compared to the pure ones as a result of the lowered symmetry. Therefore, results are shown only for the mixed compound with equal $\mathrm{Fe}$ and $\mathrm{Cr}$ concentration.

Compare the majority bands of the two pure compounds. At a first sight, the Fermi-energy is just higher in the $\mathrm{Fe}$ case compared to $\mathrm{Cr}$, as expected from the larger number of d-electrons. A closer look reveals more detailed differences. The indirect gap of the $\Delta$-direction (clearly seen for the Cr based compound) is not only shifted below $\epsilon_{F}$ but nearly closes for the Fe based compound. This gap is also nearly closed 


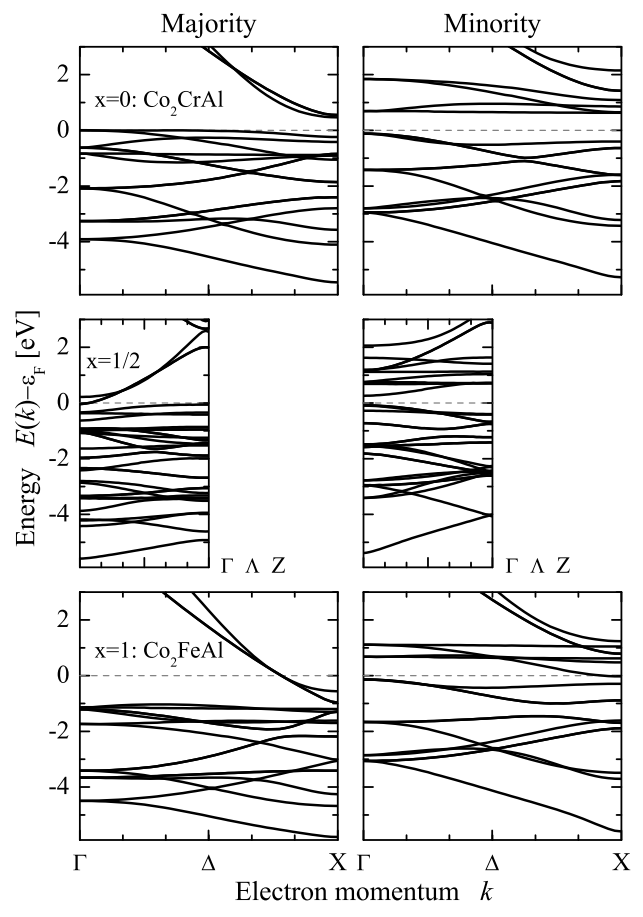

Figure 3. $\Delta, \Lambda$-bands of $\mathrm{Co}_{2} \mathrm{Cr}_{1-x} \mathrm{Fe}_{x} \mathrm{Al}$.

(The high symmetry points are $X$ for $x=0,1$ and $Z$ for $x=1 / 2$. Note that the absolute values of $k$ at $X$ are: $1.1 \AA^{-1}$ for $x=0,1$ and only $0.55 \AA^{-1}$ at $Z$ for $x=1 / 2$.)

in the majority bands of the mixed compound with $x=1 / 2$ as well as those with $x=1 / 4,3 / 4$ (not shown here). This observation calls a rigid band model in question. In that case the bands would be simply filled with increasing number of d-electrons, leaving the shape of the bands unchanged.

More interesting is the behaviour of the minority bands as those determine the HMF character of the compounds. Comparing again the $\mathrm{Cr}$ and the $\mathrm{Fe}$ based compounds, one finds that the energies of the states at $\Gamma$ are nearly the same below $\epsilon_{F}$. The shapes of the bands close to $\Gamma$ are similar, too. The situation is different at $X$ where the unoccupied states are shifted toward $\epsilon_{F}$ in $\mathrm{Co}_{2} \mathrm{FeAl}$ compared to $\mathrm{Co}_{2} \mathrm{CrAl}$.

It is worthwhile to note that the first unoccupied minority band of $\mathrm{Co}_{2} \mathrm{FeAl}$ just touches $\epsilon_{F}$ at $X$. Therefore, any temperature above $0 K$ will immediately destroy the HMF gap due to the smearing of the DOS around $\epsilon_{F}$ (for additional temperature effects destroying the minority gap see e.g.: $[56,57])$. Chioncel et al [58] reported for $\mathrm{NiMnSb}$ the occurrence of nonquasiparticle states just below the minority conduction band. A similar effect would immediately destroy the $\mathrm{HMF}$ character if appearing in $\mathrm{Co}_{2} \mathrm{FeAl}$, too. The band structure of $\mathrm{Co}_{2} \mathrm{MnAl}$ shows a similar behaviour at $\Gamma$ like $\mathrm{Co}_{2} \mathrm{FeAl}$. Here the minority bands are even crossing slightly the Fermi-energy, as was found in calculations for comparison with the iso-electronic compound $\mathrm{Co}_{2} \mathrm{Cr}_{1 / 2} \mathrm{Fe}_{1 / 2} \mathrm{Al}$. Even if accounting for small numerical deviations while calculating $\epsilon_{F}$ for the two 


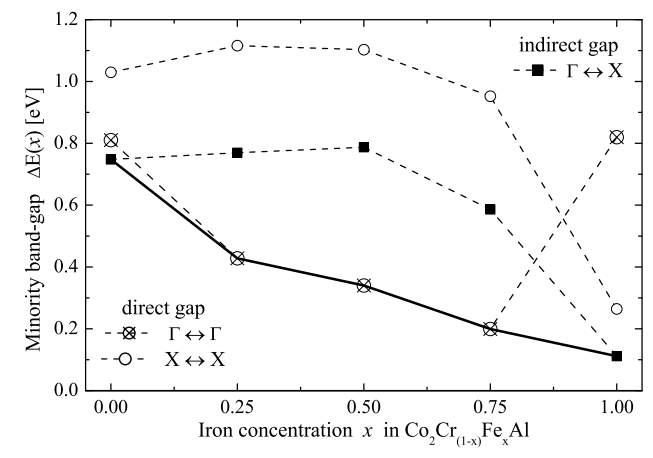

Figure 4. Minority Band Gap in $\mathrm{Co}_{2} \mathrm{Cr}_{1-x} \mathrm{Fe}_{x} \mathrm{Al}$.

(Lines are drawn to guide the eye. The full drawn line follows the limit for the HMF-gap)

compounds, they may not be good candidates for spin-injection devices.

The mixed compounds are described by $P$ lattices. Therefore, the Brillouin zone of these compounds is generally smaller compared to the $F$ lattice. This results in a seemingly back-folding of bands from the larger $F$-Brillouin zone into a smaller one. This effect is accompanied with some additional splitting (removed degeneracies) at points of high symmetry.

Due to the manifold of bands in the mixed compounds, it is not easy to compare the results directly, therefore we concentrate on the width of the gap in the minority bands. This gap is mainly characterized by the bands in $\Delta$-direction as found from the band structure for all directions of high symmetry (not shown here). More specifically, it is given by the energies at the $\Gamma$ and $X$ points of the Brillouin zone.

The width of the gap in the minority bands is shown in figure 4 . The direct band gap at the $\Gamma$-point becomes successively smaller with increasing iron content $x$ and ranges from $750 \mathrm{meV}$ at $x=0$ to $110 \mathrm{meV}$ at $x=1$. The direct gap of $\mathrm{Co}_{2} \mathrm{FeAl}$ is only $60 \mathrm{meV}$ wider than the indirect one between $\Gamma$ and $X$. The direct gap at $\Gamma$ is much wider in $\mathrm{Co}_{2} \mathrm{FeAl}$, therefore this compound is characterized by the indirect gap only.

The character of the gap changes from indirect to direct if comparing pure and mixed compounds, respectively. This change of the character of the gap in the $\Delta$ direction is a consequence of the smaller Brillouin zone in the mixed compounds that leads to a so called back-folding of bands.

The total density of states (DOS) is shown in figure 5 for varying iron content $x$. The gap at the Fermi-energy is clearly seen in the DOS of the minority states for all compounds. The total DOS shows also that the Heusler-gap at about $6 \mathrm{eV}$ binding energy is nearly closed.

The majority DOS at the Fermi energy decreases with increasing iron concentration $x$. The density of majority electrons at $\epsilon_{F}$ is a crucial point for spectroscopic methods investigating the spin polarization, like spin-resolved photoemission. A complete spin polarization may be only detectable if there is a high majority density. The same may be true for spin injection systems where one is interested in a high efficiency.

It is also seen that the minority DOS seems to be much less effected by the $\mathrm{Fe}$ 


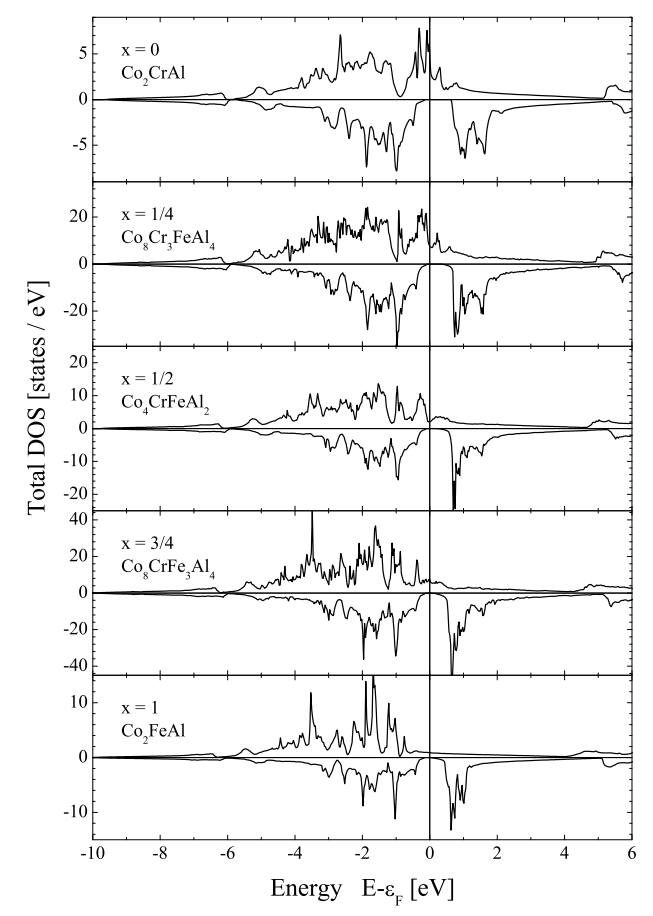

Figure 5. Total DOS of $\mathrm{Co}_{2} \mathrm{Cr}_{1-x} \mathrm{Fe}_{x} \mathrm{Al}$.

doping compared to the majority DOS. Mainly the unoccupied part of the DOS above $\epsilon_{F}$ changes its shape but not the occupied part.

In summary, it is found that doping of the compound with Fe changes mainly the occupied majority and the unoccupied minority DOS. Again it is seen that doping by iron results not just simply in a shift of the DOS as would be expected from a rigid band model. Majority and minority densities are altered in a different way.

More details of the change in the DOS and electronic structure can be extracted if analysing the partial DOS (PDOS), that is the atom type resolved density of states. The PDOS of the pure compounds is compared in figure 6 to the PDOS of the mixed compound with equal $\mathrm{Cr}$ and Fe content $(x=1 / 2)$.

From figure 6, it is seen that the high majority DOS at the Fermi-energy emerges from Cr. Both, Co and Fe exhibit only a small majority PDOS at $\epsilon_{F}$. Overall, the change of the majority DOS of $\mathrm{Co}_{2} \mathrm{Cr}_{1-x} \mathrm{Fe}_{x} \mathrm{Al}$ around $\epsilon_{F}$ can be clearly attributed to the increasing amount of iron with respect to chromium. The minimum in the minority DOS around $\epsilon_{F}$ is mainly restricted by the shape of the Co PDOS. This indicates that the HMF like behaviour is mainly characterized by Co. The steep increase of the minority PDOS of $\mathrm{Cr}$ and $\mathrm{Fe}$ is mainly located in the unoccupied part above $\epsilon_{F}$.

Doping with Fe does not only change the total DOS but also the PDOS of Co and Cr. In particular, the slight shift of the Cr PDOS to lower energies causes an additional decrease of majority states at $\epsilon_{F}$. This shift increases with increasing Fe concentration as was found from the PDOS for $x=1 / 4$ and $3 / 4$ (not shown here). The slight energy shift of the PDOS will result in a small change of the local magnetic 

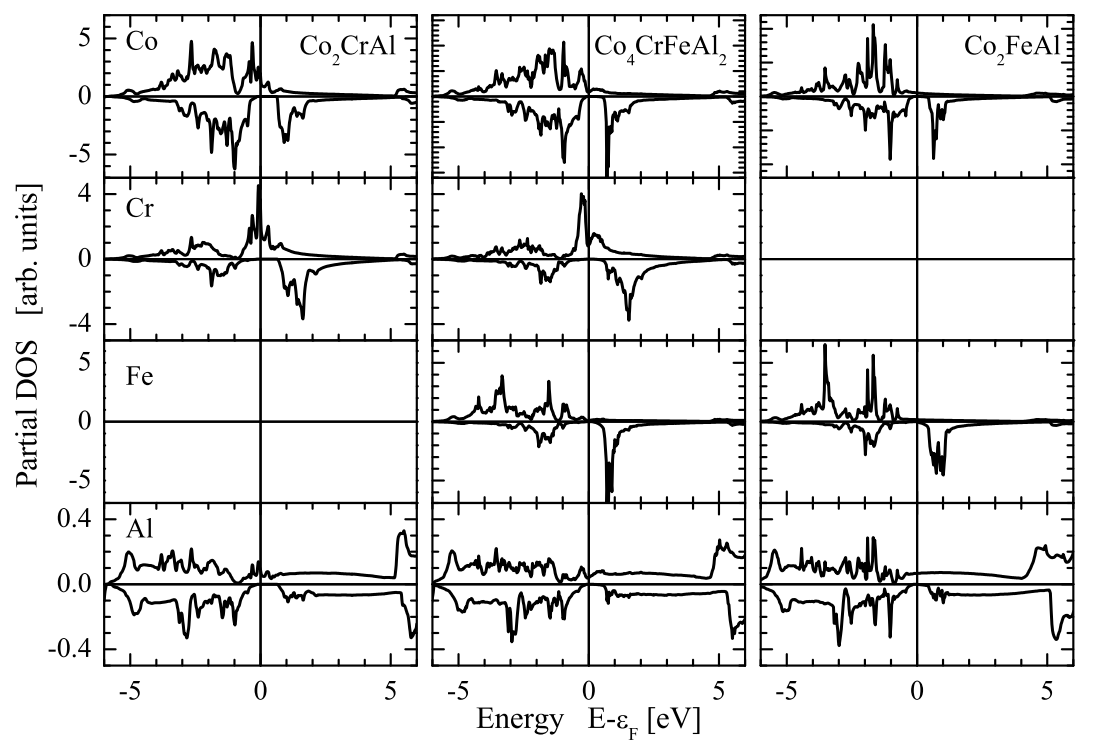

Figure 6. Partial DOS of $\mathrm{Co}_{2} \mathrm{Cr}_{1-x} \mathrm{Fe}_{x} \mathrm{Al}(x=0,1 / 2,1)$.

moments at the Co and $\mathrm{Cr}$ sites, as will be shown below.

The aluminium PDOS stays rather unaffected from the $\mathrm{Cr}$ or Fe concentration. It exhibits only small energy shifts.

\subsection{Magnetic moments}

SQUID magnetometry and MCD were used to determine total and partial magnetic moments of $\mathrm{Co}_{2} \mathrm{Cr}_{1-x} \mathrm{Fe}_{x} \mathrm{Al}$. Details of these measurements are reported in Ref. [46]. The measured values are extrapolated to $T=4 K$ and calibrated using the total moments measured by SQUID. Total and element specific magnetic moments were extracted from the band structure calculations reported above. Figure 7 compares measured and calculated spin magnetic moments.

The calculated total spin magnetic moment follows the rule of thumb for Heusler compounds:

$$
\mu_{s}=(N-24) \mu_{B}
$$

$N$ is the cumulated number of valence electrons (here: $4 \mathrm{~s}, 3 \mathrm{~d}$ for the transition metals $\mathrm{Co}, \mathrm{Cr}$, or $\mathrm{Fe}$ and $3 \mathrm{~s}, 3 \mathrm{p}$ for the main group element $\mathrm{Al}$ ). The value calculated from equation 1 is shown as full line in figure $7 \mathrm{a}$.

The calculated spin magnetic moments of $\mathrm{Co}$ and $\mathrm{Fe}$ are in agreement with the measured values. The measured, lower total value at small Fe concentration can be attributed clearly to a too low moment at the Cr sites.

The calculated spin moments of $\mathrm{Co}$ and $\mathrm{Cr}$ increase slightly with increasing Fe concentration, whereas the Fe moment stays nearly constant. This increase is explained by an energy shift of the partial densities of $\mathrm{Co}$ and $\mathrm{Cr}$ as discussed above. 


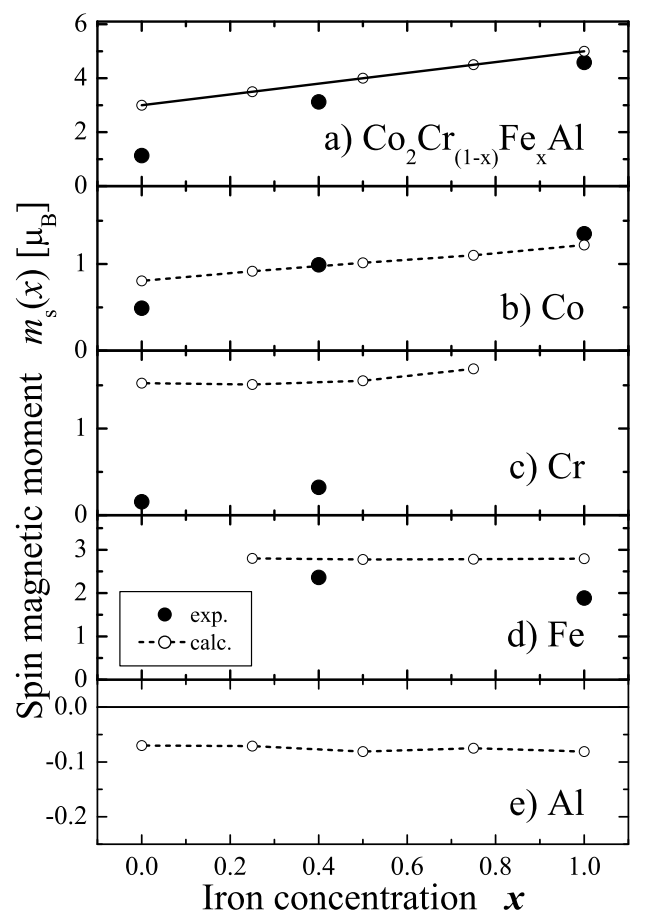

Figure 7. Magnetic moments of $\mathrm{Co}_{2} \mathrm{Cr}_{1-x} \mathrm{Fe}_{x} \mathrm{Al}$.

The measured element specific and total moments for $(x=0,0.4$, and 1) are compared to calculated values for $(x=0,1 / 4,1 / 2,3 / 4$, and 1$)$. The full line in a) corresponds to the thumb-rule, dashed lines in b)...e) are drawn through the calculated values to guide the eye.

The calculated $\mathrm{Al}$ spin moment is negative, independent of the Fe concentration. This points on an anti-ferromagnetic order of the $\mathrm{Al}$ moments with respect to the transition metal moments. However, those induced moments at the $\mathrm{Al}$ sites are only very small.

All values found here for the pure compounds are in the same order of those calculated by Galanakis et.al. [28] using the Korringa-Kohn-Rostokker (KKR) method.

It may be interesting to compare the magnetic moments of $\mathrm{Co}_{2} \mathrm{Cr}_{0.5} \mathrm{Fe}_{0.5} \mathrm{Al}$ with those of the nominally iso-electronic compound $\mathrm{Co}_{2} \mathrm{MnAl}$. Our calculations yielded $3.81 \mu_{B}$ for the total spin moment per formula unit and $0.84 \mu_{B}$ and $2.58 \mu_{B}$ for the partial spin moments of $\mathrm{Co}$ and $\mathrm{Mn}$, respectively. These values are similar to those found by other groups $[23,28]$ using KKR. The Co moment is larger in the mixed compound $\left(1.01 \mu_{B}\right)$ and the average $\mathrm{Cr}_{0.5} \mathrm{Fe}_{0.5}$ moment $\left(2.24 \mu_{B}\right)$ is smaller compared to $\mathrm{Mn}$. It is interesting to note that the minority states were shifted at the $\Gamma$-point to energies above $\epsilon_{F}$, such that the HMF-gap became closed in $\mathrm{Co}_{2} \mathrm{MnAl}$. A variation of the lattice parameters showed that this is not causing the differences between the two materials. The differences are caused by the different local potentials in those compounds.

Most evidently, the calculated magnetic moment of the $\mathrm{Co}_{2} \mathrm{CrAl}$ compound does 
Table 2. Magnetic moments in $\mathrm{Co}_{2} \mathrm{CrAl}$. Element specific (per atom) and total spin magnetic moments (per formula unit) calculated by different methods.

\begin{tabular}{lccc} 
& \multicolumn{3}{c}{$m_{s}\left[\mu_{B}\right]$} \\
Method & Co & Cr & tot \\
\hline LMTO-ASA & 0.67 & 1.72 & 2.98 \\
LMTO-ASA-GGA & 0.70 & 1.63 & 2.98 \\
FP-LMTO-PLW & 0.73 & 1.61 & 3.01 \\
FLAPW-GGA & 0.81 & 1.52 & 3.00 \\
KKR [28] & 0.76 & 1.54 & 2.96
\end{tabular}

not agree with the measured one. The experimental value is only about $1.3 \mu_{B}$ whereas theory predicts a value of about $3 \mu_{B}$ per formula unit. The value found here is in rough agreement to the ground state magnetic moment of $1.55 \mu_{B}$ per formula unit reported earlier by Buschow [59]. It was previously considered that mainly the Co atoms carry the magnetic moment, whereas the contribution of $\mathrm{Cr}$ and $\mathrm{Al}$ atoms remains negligible [60]. This empiric assumption may describe the present element specific measurements revealing a moment of $\approx 2 \times 0.55 \mu_{B}$ for $\mathrm{Co}_{2}$ but only $\approx 0.2 \mu_{B}$ for Cr. However, it does not explain the physics behind that observation.

One may assume that the high magnetic moment of $\mathrm{Cr}$ is an artefact of a particular calculation scheme. Therefore, different calculation schemes were used to check a probable occurrence of such effects. The results are summarized in table 2. The results of Galanakis et al [28] derived from KKR-calculations are shown for comparison. The partial and total magnetic moments calculated by Kellou et al [61] at a smaller lattice parameter $\left(10.758 a_{0 B}\right)$ are in the same range.

It is seen from table 2 that all values stay comparable within few $\%$. Therefore, the deviation from the experiment cannot be attributed to the peculiarities of one or another theoretical method. The $\mathrm{Cr}$ moment is about $(1.6 \pm 0.1) \mu_{B}$ rather independent of the method of calculation.

On the other hand, a reduction of the observed $\mathrm{Cr}$ moment may be caused by site-disorder, such that part of the $\mathrm{Cr}$ atoms become anti-ferromagnetically ordered either among each other or with respect to the Co atoms. The latter results in a ferrimagnetic order. A mixture of ordered and disordered crystallites will then result in a measured value being too small compared to the calculated moment. Such a disorder has to be realized with the same lattice parameter because X-ray diffraction did not show any superstructure in the samples investigated here.

To estimate the influence of disorder in the CrAl-planes, the band structure was also calculated for the hypothetical $c P 16$ ferrite structure $(P m \overline{3} m)$. In this structure, successive $\mathrm{CrAl}-(001)$-planes are $\mathrm{Cr}$ or $\mathrm{Al}$ rich with $3: 1$ or $1: 3$ ratios, respectively. The calculated magnetic moments of $\mathrm{Cr}$ were about $1.5 \mu_{B}$. Lin and Freeman [62] used the tetragonal $t P 4$ structure $(P 4 / \mathrm{mmm})$ to simulate a Y/Al disorder in $\mathrm{Ni}_{2} \mathrm{YAl}$ $(\mathrm{Y}=\mathrm{Ti}, \mathrm{V}$, etc.). In this structure one has the series $\mathrm{Co}-\mathrm{Cr}-\mathrm{Co}-\mathrm{Al}$ for the consecutive (001)-planes instead of $\mathrm{Co}-(\mathrm{CrAl})$ (Note that (100) and (001) planes are not equivalent in the tetragonal structure). Again, the calculated magnetic moment of $\mathrm{Cr}$ has about the same magnitude. A ground state with an anti-ferromagnetic or ferrimagnetic order of the $\mathrm{Cr}$ atoms could not be verified in either of both structures. Therefore, those 
Table 3. Structural dependence of the magnetic moments in $\mathrm{Co}_{2} \mathrm{CrAl}$. Note: The sum of the magnetic moments of the two individual Co atoms and average values for $\mathrm{Cr}$ and $\mathrm{Al}$ are given to make different structures comparable. The total spin magnetic moment is given per formula unit.

\begin{tabular}{llcccc} 
& & & \multicolumn{3}{c}{$m\left[\mu_{B}\right]$} \\
& Structure & $\mathrm{Co}_{2}$ & $\mathrm{Cr}$ & $\mathrm{Al}$ & tot \\
\hline$L 2_{1}$ & $F m \overline{3} m$ & 1.42 & 1.63 & -0.05 & 2.98 \\
$X$ & $F \overline{4} 3 m$ & 1.84 & -0.83 & -0.02 & 0.91 \\
$c P 16$ & $P m \overline{3} m$ & 1.69 & 1.48 & -0.05 & 3.00 \\
$t P 4$ & $P 4 / m m m$ & 1.82 & 1.47 & -0.05 & 3.19 \\
& Experiment & 1.11 & 0.19 & - & 1.30
\end{tabular}

structure can be excluded as possible candidates for explanation of the reduction of the magnetic moment. This is in accordance with the X-ray diffraction data where no additional Bragg peaks were observed. Extra (001) Bragg peaks are expected for both structures, for example. However, it is worthwhile to note that the compound still exhibited HMF character in the $c P 16$ structure.

More promising are calculations for the $X$-structure $(F \overline{4} 3 m)$ [63]. (Note that the Pearson code of this structure is $c F 16$ like for $L 2_{1}$.) This structure was also considered among several phases with random anti-site disorder by Feng et al [64] investigating the physical properties of $\mathrm{Fe}_{2} \mathrm{VAl}$. It is similar to the $C 1_{b}$ Half-Heusler phase but with the vacancies filled up in a different way compared to the $L 2_{1}$ structure. The $X$-structure consists of successive $\mathrm{CoCr}$ and $\mathrm{CoAl}$ (001)-planes.

The calculation reveals for the $X$-structure an anti-ferromagnetic ordering of the $\mathrm{Cr}$ atoms with respect to $\mathrm{Co}$ and thus a reduced overall magnetic moment. The magnetic moments derived for the different structures are summarized in table 3 .

The calculated, element specific and total magnetic moments are compared to experimental values in table 3 . For Co the sum of both atoms is given. The average of the calculated values for $\mathrm{Cr}$ and $\mathrm{Al}$ are given for the structures with inequivalent atomic positions.

A more detailed analysis of the $X$-structure shows not only an anti-ferromagnetic order of $\mathrm{Cr}$ but also an enhancement of the Co magnetic moments. Their values are $0.94 \mu_{B}$ in $\mathrm{CoAl}$ and $0.76 \mu_{B}$ in $\mathrm{CoCr}(001)$-planes. Evidently the shortest distance between two Co atoms is smaller in the $X$-structure compared to the $L 2_{1}$-structure (see table 4). The shortest $\mathrm{Co}-\mathrm{Cr}$ and $\mathrm{Cr}-\mathrm{Cr}$ distances stay the same in both structures.

The enhancement of the Co moments in all three non- $L 2_{1}$ structures does not allow to explain the experimentally observed moments directly because the measured value is already smaller as the calculated moment of the $L 2_{1}$ structure. This is mainly a property of the particular sample investigated here. Other samples exhibited higher overall magnetic moments.

The gap in the minority band structure is closed in the $X$-structure, that means it is not longer a half-metallic ferromagnet. The vanishing of the gap was also found for $\mathrm{Co}_{2} \mathrm{FeAl}$ in the $X$-structure, but with the $\mathrm{Fe}$ atoms aligned ferromagnetically with respect to Co. It is interesting to note that the $c P 16$ structure still exhibits the gap for both pure compounds. From this observation it can be concluded that the existence of the gap is directly related to the occurrence of $\mathrm{Co}_{2}$ and mixed $\mathrm{CrAl}$ (001)-planes and that the $L 2_{1}$-structure is not the only possibility for the presence of a HMF gap 
in Heusler-like compounds $\mathrm{X}_{2} \mathrm{YZ}$.

This finding can be understood easily considering symmetry. The magnetization will reduce the symmetry. Applying any special orientation along one of the principal axes (e.g.: [001]) will reduce the symmetry from $F m \overline{3} m$ to $I 4 / m m m$, at least. This is in accordance with the fact that the $O_{h}$ point group cannot describe ferromagnetic order. The properties of the electron spin will cause a further reduction to $I 4 / m$, as vertical mirror operations would change the sign of the spin. In particular, the $\Gamma$-point of the ferromagnetic Heusler compounds will belong to the $D_{4 h}\left(C_{4 h}\right)$ colour group and not longer to $O_{h}$ like in the paramagnetic state. The point group in brackets assigns the magnetic symmetry with removed vertical mirror planes. The $X$-point becomes $Z$ $\left(D_{4 h}\left(C_{4 h}\right)\right)$ and the point group symmetry of the $\Lambda$-direction (formerly $\Delta$ ) is reduced to $C_{4 v}\left(C_{4}\right)$ in the ferromagnetic case.

The $\Gamma$ and $Z$ points of the $c P 16$ or $t P 4$ structures, as representatives for $\mathrm{Cr}-\mathrm{Al}$ disorder, have in the ferromagnetic state also $D_{4 h}\left(C_{4 h}\right)$ symmetry. Therefore, those structures are expected to behave similar like the $L 2_{1}$-structure. However, the local symmetry of the atomic sites in the three structures is different what may explain the vanishing of the HMF gap in the $t P 4$ structure.

The $\Gamma$ and $Z$ points of the $X$-structure, as representative for $\mathrm{Co}-\mathrm{Cr}$ anti-site disorder, have in the ferromagnetic state the lower $D_{2 d}\left(S_{4}\right)$ point group symmetry. Therefore, that structure is expected to behave different from the $L 2_{1}$-structure. Indeed, the most pronounced difference is the local environment of the atoms in the (001)-planes.

The local site symmetries of the atoms are summarized in table 4 . It displays the symmetries of atoms and high symmetric points of the Brillouin-zone for the different structures for paramagnetic (PM) and ferromagnetic (FM) order. The direction of magnetization was chosen to be along the $z$-axis for $\mathrm{FM}$ order. $\mathrm{Cr}$ and $\mathrm{Al}$ atoms occupy in the $c P 16$ structure two inequivalent sites with different local symmetry. The distances between various atoms are given for a fixed lattice parameter $a$. The $X$-point becomes $Z$ and the $\Delta$-direction becomes $\Lambda$ in tetragonal symmetry. The point group symmetries of the Brillouin-zone may serve to avoid confusion about the irreducible representation of bands being different in the PM and FM state.

The $\mathrm{Cr}$ magnetic moment is also too small in the mixed $\mathrm{Co}_{2} \mathrm{Cr}_{1-x} \mathrm{Fe}_{x} \mathrm{Al}$ alloy, as seen from figure 7 . The Fe magnetic moment comes close to the value expected from the calculation. This observation points not only on a site-disorder but also on the possibility of phase separation resulting in $\mathrm{Fe}$ and $\mathrm{Cr}$ rich grains of the polycrystalline sample. The overall magnetic moment comes close to the calculated value as result of the much higher value for Fe compared to Cr. From this observation it is clear that measuring only the total moment is not enough to characterize these alloys completely.

Similar calculations were performed for the Half-Heusler compound NiMnSb in order to explain a missing full spin polarization in that compound. In the XYZ $C 1_{b}$-structure one has a series of pure $\mathrm{X}$ followed by mixed YZ (001)-planes. The calculations were performed for NiMnSb, MnSbNi, and SbNiMn, keeping the lattice parameter fixed. The major difference between the three types is the local environment of the $\mathrm{Ni}$ atoms in the (001) planes. Only in the first type, the alternating (001)planes contain either purely $\mathrm{Ni}$ or mixed $(\mathrm{MnSb})$ layers. The exchange of $\mathrm{Ni}$ and $\mathrm{Mn}$ or $\mathrm{Sb}$ resulted in the loss of the HMF character and in turn in a reduction of the spin polarization at the Fermi-energy. Indeed, such an intermixing must not prevail through the hole crystal. It is just enough to have disorder in regions close to the surface or interface in order to explain a reduced spin polarization in spectroscopic 
Table 4. Site symmetries in $\mathrm{Co}_{2} \mathrm{CrAl}$.

$\mathrm{PM}:=$ paramagnetic order, $\mathrm{FM}:=$ ferromagnetic order with $B \|[001]$. The nearest distance $d_{A B}$ between two atoms $\mathrm{A}$ and $\mathrm{B}$ is given in $\AA$. The second and $4^{t h}$ lines for each structure give the Wyckoff positions of the atoms in the PM and FM state.

\begin{tabular}{|c|c|c|c|c|c|c|c|c|c|c|c|c|}
\hline & & & Co & $\mathrm{Cr}$ & $\mathrm{Al}$ & $d_{\text {CoCo }}$ & $d_{C o C r}$ & $d_{C r C r}$ & $d_{C r A l}$ & $\Gamma$ & $\Delta,(\Lambda)[0,0, \mathrm{z}]$ & $X,(Z)$ \\
\hline \multirow[t]{2}{*}{$\begin{array}{l}L 2_{1} \\
c F 16\end{array}$} & $\mathrm{PM}$ & $F m \overline{3} m$ & $\begin{array}{l}T_{d} \\
8 \mathrm{c}\end{array}$ & $\begin{array}{l}O_{h} \\
4 \mathrm{a}\end{array}$ & $\begin{array}{c}O_{h} \\
4 \mathrm{~b}\end{array}$ & 2.86 & 2.48 & 4.05 & 2.86 & $O_{h}$ & $C_{4 v}$ & $D_{4 h}$ \\
\hline & $\mathrm{FM}$ & $I 4 / m$ & $\begin{array}{l}S_{4} \\
4 \mathrm{~d}\end{array}$ & $\begin{array}{c}C_{4 h} \\
2 \mathrm{a}\end{array}$ & $\begin{array}{c}C_{4 h} \\
2 \mathrm{~b}\end{array}$ & & & & & $D_{4 h}\left(C_{4 h}\right)$ & $C_{4 v}\left(C_{4}\right)$ & $D_{4 h}\left(C_{4 h}\right)$ \\
\hline \multirow[t]{2}{*}{$\begin{array}{l}X \\
c F 16\end{array}$} & PM & $F \overline{4} 3 m$ & $\begin{array}{c}T_{d} \\
4 \mathrm{a}, 4 \mathrm{c}\end{array}$ & $\begin{array}{l}T_{d} \\
4 \mathrm{~b}\end{array}$ & $\begin{array}{l}T_{d} \\
4 \mathrm{~d}\end{array}$ & 2.48 & 2.48 & 4.05 & 2.48 & $T_{d}$ & $C_{2 v}$ & $D_{2 d}$ \\
\hline & FM & $I \overline{4}$ & $\begin{array}{c}S_{4} \\
2 \mathrm{a}, 2 \mathrm{~d} \\
\end{array}$ & $\begin{array}{l}S_{4} \\
2 \mathrm{~b}\end{array}$ & $\begin{array}{l}S_{4} \\
2 \mathrm{c}\end{array}$ & & & & & $D_{2 d}\left(S_{4}\right)$ & $C_{2 v}\left(C_{2}\right)$ & $D_{2 d}\left(S_{4}\right)$ \\
\hline \multirow[t]{2}{*}{$c P 16$} & PM & & $\begin{array}{c}C_{3 v} \\
8 \mathrm{~g}\end{array}$ & $\begin{array}{c}O_{h}, D_{4 h} \\
1 \mathrm{a}, 3 \mathrm{~d}\end{array}$ & $\begin{array}{c}D_{4 h}, O_{h} \\
3 \mathrm{c}, 1 \mathrm{~b}\end{array}$ & 2.86 & 2.86 & 2.48 & 2.86 & $O_{h}$ & $C_{4 v}$ & $D_{4 h}$ \\
\hline & FM & $P 4 / m$ & $\begin{array}{c}C_{1} \\
81 \\
\end{array}$ & $\begin{array}{c}C_{4 h}, C_{2 h}, C_{2 h} \\
1 \mathrm{a}, 2 \mathrm{e}, 1 \mathrm{~b} \\
\end{array}$ & $\begin{array}{c}C_{2 h}, C_{2 h}, C_{4 h} \\
1 \mathrm{c}, 2 \mathrm{f}, 1 \mathrm{~d} \\
\end{array}$ & & & & & $D_{4 h}\left(C_{4 h}\right)$ & $C_{4 v}\left(C_{4}\right)$ & $D_{4 h}\left(C_{4 h}\right)$ \\
\hline \multirow[t]{2}{*}{$t P 4$} & PM & $P 4 / m m m$ & $\begin{array}{l}C_{4 v} \\
2 \mathrm{~h}\end{array}$ & $\begin{array}{c}D_{4 h} \\
1 \mathrm{a}\end{array}$ & $\begin{array}{c}D_{4 h} \\
1 \mathrm{~b}\end{array}$ & 2.86 & 2.48 & 2.86 & 2.86 & $D_{4 h}$ & $C_{4 v}$ & $D_{4 h}$ \\
\hline & FM & $P 4 / m$ & $\begin{array}{l}C_{4} \\
2 \mathrm{~h}\end{array}$ & $\begin{array}{c}C_{4 h} \\
1 \mathrm{a}\end{array}$ & $\begin{array}{c}C_{4 h} \\
1 \mathrm{~b}\end{array}$ & & & & & $D_{4 h}\left(C_{4 h}\right)$ & $C_{4 v}\left(C_{4}\right)$ & $D_{4 h}\left(C_{4 h}\right)$ \\
\hline
\end{tabular}


methods. These findings are in agreement with the results of Orgassa et al [65] for random disorder in NiMnSb.

The assumption of doped, ordered compounds may not hold in every case, especially if considering non half- or non quarter-integer fractions for the iron concentration $x$. Calculations concerning the spectroscopic properties for more general, non-rational iron concentration, resulting in random alloys, are in preparation. However, the results found here for superstructures are in well agreement to those of Miura et al [47] for random alloys.

\section{Summary}

The electronic structure of the pure and doped Heusler compound $\mathrm{Co}_{2} \mathrm{Cr}_{1-x} \mathrm{Fe}_{x} \mathrm{Al}$ with varying iron content $(x)$ was calculated by means of different theoretical methods. Element specific magnetic moments were determined from MCD measurements at the $\mathrm{Cr}, \mathrm{Fe}$, and $\mathrm{Co} L_{2,3}$ absorption edges of the Heusler alloys $\mathrm{Co}_{2} \mathrm{CrAl}, \mathrm{Co}_{2} \mathrm{Cr}_{0.6} \mathrm{Fe}_{0.4} \mathrm{Al}$, and $\mathrm{Co}_{2} \mathrm{FeAl}$.

The calculations revealed a ferromagnetic coupling between the $3 d$ atoms as well as an anti-ferromagnetic alignment of the $\mathrm{Al}$ magnetic moments with respect to the moments of the $3 d$ elements. However, the $\mathrm{Al}$ moments are very small and induced by the surrounding polarized atoms. The calculations predict the $\mathrm{Co}_{2} \mathrm{Cr}_{1-x} \mathrm{Fe}_{x} \mathrm{Al}$ compound to be a half-metallic ferromagnet. The size of the minority gap ranges from $100 \mathrm{meV}$ to $800 \mathrm{meV}$. The smallest band-gap around the Fermi-edge in the minority bands was found for the compound with $x=1 . \mathrm{Co}_{2} \mathrm{CrAl}$ and, more pronounced, $\mathrm{Co}_{2} \mathrm{FeAl}$ turned out to have an indirect $\Gamma-X$ gap. The mixed compounds exhibit a direct gap at the $\Gamma$ point being caused by the reduced symmetry.

It was shown that the origin of the minority gap in $\mathrm{Co}_{2} \mathrm{CrAl}$ is the geometrical structure and local symmetry of the atoms. It appears in the $L 2_{1}$-structure with successive $\mathrm{CrAl}$ and $\mathrm{Co}_{2}$ (001)-planes but not in the $X$-structure with successive $\mathrm{CoCr}$ and $\mathrm{CoAl}(001)$-planes.

In summary, it was shown how theoretical methods can be used to design new materials with predictable magnetic properties.

\section{Acknowledgments}

The authors thank all members of NSRRC (Hsinchu, Taiwan) for their help during the beamtimes. G.H.F. and S.W. are very grateful to Yeukuang Hwu (Academia Sinica, Taipei) and his group for support during the experiments in Taiwan.

Financial support by the DFG (FG 559), DAAD (03/314973 and 03/23562), and PROBRAL (167/04) is gratefully acknowledged. 
[1] G. A. Prinz. Science, 282:1660, 1998.

[2] R. A. de Groot, F. M. Mueller, P. G. van Engen, and K. H. J. Buschow. Phys. Rev. Lett., 50:2024, 1983.

[3] J. M. D. Coey, M. Venkatesan, and M. A. Bari. Half-metallic ferromagnets. In C. Berthier, L. P. Levy, and G. Martinez, editors, Lecture Notes in Physics, volume 595, pages $377-396$. Springer-Verlag, Heidelberg, 2002.

[4] S. J. Youn and B. I. Min. Phys. Rev. B, 51:10436 - 10442, 1995.

[5] D. Ristoiu, J. P. Nozieres, C. N. Borca, B. Borca, and P. A. Dowben. Appl. Phys. Lett., 76:2349, 2000.

[6] D. Ristoiu, J. P. Nozieres, C. N. Borca, T. Komesu, H.-K. Jeong, and P. A. Dowben. Eur. Phys. Lett., 49:624-630, 2000.

[7] W. Zhu, B. Sinkovic, E. Vescovo, C. Tanaka, and J. S. Moodera. Phys. Rev. B, 64:R060403, 2001.

[8] S. Plogmann, T. Schlathölter, J. Braun, M. Neumann, Y. M. Yarmoshenko, M. V. Yablonskikh, E. I. Shreder, E. Z. Kurmaev, A. Wrona, and A. Slebarski. Phys. Rev. B, 60:6428, 1999.

[9] P. J. Webster and K. R. A. Ziebeck. J. Phys. Chem. Solids, 34:1647, 1973.

[10] Fr. Heusler. Verh. Dtsch. Phys. Ges., 12:219, 1903.

[11] M. Terada, Y. Fujita, and K. Endo. J. Phys. Soc. Jpn., 36:620, 1974.

[12] K. Endo, K. Ooiwa, and A. Shinogi. J. Magn. Magn. Mater., 104 - 107:2013 - 2014, 1992.

[13] K. R. A. Ziebeck and P. J. Webster. J. Phys. Chem. Solids, 35:1, 1974.

[14] P. G. van Engen, K. H. J. Buschow, and M. Erman. J. Magn. Magn. Mater., 30:374 - 382, 1983.

[15] M. V. Yablonskikh, Y. M. Yarmoshenko, V. I. Grebennikov, E. Z. Kurmaev, S. M. Butorin, L.-C. Duda, J. Nordgren, S. Plogmann, and M. Neumann. Phys. Rev. B, 63:235117, 2001.

[16] S. Ishida, S. Akazawa, Y. Kubo, and J. Ishida. J. Phys. F: Met. Phys., 12:1111 - 1122, 1982.

[17] P. J. Webster and K. R. A. Ziebeck. Heusler alloys. In Alloys and Compounds of d-Elements with Main Group Elements. Part 2, volume 19C of Landolt-Börnstein - Group III Condensed Matter, pages 104 - 185. Springer-Verlag, Heidelberg, 1988.

[18] K. R. A. Ziebeck and K.-U. Neumann. Heusler alloys. In Alloys and Compounds of d-Elements with Main Group Elements. Part 2, volume 38C of Landolt-Börnstein - Group III Condensed Matter, pages 64 - 314. Springer-Verlag, Heidelberg, 2001.

[19] A. Jezierski. J. Magn. Magn. Mater., 164:381 - 384, 1996.

[20] P. J. Brown, K.-U. Neumann, P. J. Webster, and K. R. A. Ziebeck. J. Phys.: Condens. Matter, 12:1827 - 1835, 2000.

[21] A. Yamasaki, S. Imada, R. Arai, H. Utsunomiya, S. Suga, T. Muro, Y. Saito, T. Kanomata, and S. Ishida. Phys. Rev. B, 65:104410, 2002.

[22] S. Ishida, Y. Otsuka, Y. Kubo, and J. Ishida. J. Phys. F: Met. Phys., 13:1173 - 1178, 1983.

[23] S. Fuji, S. Sugimura, S. Ishida, and S. Asano. J. Phys.: Condens. Matter, 2:8583 - 8589, 1990.

[24] A. W. Carbonari, R. N. Saxena, Jr. W. Pendl, J. Mestnik Filho, R. N. Attili, M. Olzon-Dionysio, and S. D. de Souza. J. Magn. Magn. Mater., 163:313 - 321, 1996.

[25] S. Ishida, T. Masakai, S. Fujii, and S. Asano. Physica B, 245:1 - 8, 1998.

[26] A. Slebarski, A. Jezierski, S. Lütkehoff, and M. Neumann. Phys. Rev. B, 57:6408 - 6412, 1998.

[27] S. Picozzi, A. Continenza, and A. J. Freeman. Phys. Rev. B, 66:094421, 2002.

[28] I. Galanakis, P. H. Dederichs, and N. Papanikolaou. Phys. Rev. B, 66:174429, 2002.

[29] A. Jezierski and G. Borstel. Physica B, 205:397 - 402, 1995.

[30] A. Slebarski, A. Jezierski, M. Neumann, and S. Plogmann. J. Magn. Magn. Mater., 185:43 48, 1998.

[31] A. Slebarski, E. Talik, G. Chelkowska, and A. Jezierski. J. All. Comp., 287:45 - 47, 1999.

[32] C. S. Lue and Y.-K. Kuo. Phys. Rev. B, 66:085121, 2002.

[33] M. Pugaczowa-Michalska. phys. stat. sol. (b), 236:536 - 539, 2003.

[34] T. Block, C. Felser, G. Jakob, J. Ensling, B. Mühling, P. Gütlich, V. Beaumont, F. Studer, and R. J. Cava. J. Solid State Chem., 176:646 - 651, 2003.

[35] C. Felser, B. Heitkamp, F. Kronast, D. Schmitz, S. Cramm, H. A. Dürr, H.-J. Elmers, G. H. Fecher, S. Wurmehl, T. Block, D. Valdaitsev, S. A. Nepijko, A. Gloskovskii, G. Jakob, G. Schönhense, and W. Eberhardt. J. Phys.: Condens. Matter, 15:7019 - 7027, 2003.

[36] R. Kelekar and B. M. Clemens. J. Appl. Phys., 96:540, 2004.

[37] A. Hirohata, M. Kikuchi, N. Tezuka, K. Inomata, J. S. Claydon, and Y. B. Xu. J. Appl. Phys., 97:10C308, 2005.

[38] A. Hirohata, H. Kurebayashi, S. Okamura, M. Kikuchi, T. Masaki, T. Nozaki, N. Tezuka, and K. Inomata. J. Appl. Phys., 97:103714, 2005.

[39] G. Jakob, F. Casper, V. Beaumont, S. Falka, N. Auth, H.-J. Elmers, C. Felser, and H. Adrian. 
Design of magnetic materials: $\mathrm{Co}_{2} \mathrm{Cr}_{1-x} \mathrm{Fe}_{x} \mathrm{Al}$.

J. Magn. Magn. Mat., 290-291:1104 - 1107, 2005.

[40] K. Inomata, S. Okamura, R. Goto, and N. Yezuka. Jpn. J. Appl. Phys., 42:L419, 2003.

[41] K. Inomata, N. Tezuka, S. Okamura, H. Kurebayashi, and A. Hirohata. J. Appl. Phys., 95:7234, 2004.

[42] T. Marukame, T. Kasahara, K.-I. Matsuda, T. Uemura, and M. Yamamoto. Jpn. J. Appl. Phys., 44:L521 - L524, 2005.

[43] N. Auth, G. Jakob, T. Block, and C. Felser. Phys. Rev. B, 62:024403, 2003.

[44] A. Conca, S. Falk, G. Jakob, M. Jourdan, and H. Adrian. J. Magn. Magn. Mat., 290-291:1127 $-1130,2005$.

[45] E. Clifford, M. Venkatesan, R. Gunning, and J. M. D. Coey. Solid State Comm., 131:61 - 64, 2004.

[46] H.-J. Elmers, G. H. Fecher, D. Valdaitsev, S. A. Nepijko, A. Gloskovskii, G. Jakob, G. Schönhense, S. Wurmehl, T. Block, C. Felser, P.-C. Hsu, W.-L. Tsai, and S. Cramm. Phys. Rev. B, 67:104412, 2003.

[47] Y. Miura, K. Nagao, and M. Shirai. Phys. Rev. B, 69:144413, 2004.

[48] K. Kobayashi, R. Y. Umetsu, R. Kainuma, K. Ishida, T. Oyamada, A. Fujita, and K. Fukamichi. Appl. Phys. Lett., 85:4684, 2004.

[49] P. Blaha, K. Schwarz, P. Sorantin, and S. B. Tricky. Comput. Phys. Commun., 59:399, 1990.

[50] P. Blaha, K. Schwarz, G. K. H. Madsen, D. Kvasnicka, and J. Luitz. WIEN2k, An Augmented Plane Wave + Local Orbitals Program for Calculating Crystal Properties. Karlheinz Schwarz, Techn. Universitaet Wien, Wien, Austria, 2001.

[51] J. P. Perdew, J. A. Chevary, S. H. Vosko, K. A. Jackson, M. R. Pederson, D. J. Singh, and C. Fiolhais. Phys. Rev. B, 46:6671, 1992.

[52] S. Y. Savrasov. Phys. Rev. B 54, 54:16470, 1996.

[53] J. Tobola, J. Pierre, S. Kaprzyk, R. V. Skolozdra, and M. A. Kouacou. J. Magn. Magn. Mater., 159:192 - 200, 1996.

[54] R. A. de Groot, F. M. Mueller, P. G. van Engen, and K. H. J. Bushow. J. Appl. Phys., 55:2151, 1984.

[55] S. Ögüt and K. M. Rabe. Phys. Rev. B, 51:10443 - 10453, 1995.

[56] R. Skomski and P. A. Dowben. The finite-temperature densities of states for half-metallic ferromagnets. Europhys. Lett., 58:544-548, 2002.

[57] P. A. Dowben and R. Skomski. J. Appl. Phys., 93:7948, 2003.

[58] L. Chioncel, M. I. Katsnelson, R. A. de Groot, and A. I. Lichtenstein. Phys. Rev. B, 68:144425, 2003.

[59] K. H. J. Buschow and P. G. van Engen. J. Magn. Magn. Mater., 25:90 - 96, 1981.

[60] A. Slebarski, M. Neumann, and B. Schneider. J. Phys.: Condens. Matter, 13:5515 - 5518, 2001.

[61] A. Kellou, N. E. Fenineche, T. Grosdidier, H. Aourag, and C. Coddet. J. Appl. Phys., 94:3292, 2003.

[62] W. Lin and A. J. Freeman. Phys. Rev. B, 45:61, 1992.

[63] G. E. Bacon and J. S. Plant. J. Phys. F: Met. Phys., 1:524 - 532, 1971.

[64] Y. Feng, J. Y. Rhee, T. A. Wiener, D. W. Lynch, B. E. Hubbard, A. J. Sievers, D. L. Schlagel, T. A. Lograsso, and L. L. Miller. Phys. Rev. B, 63:165109, 2001.

[65] D. Orgassa, H. Fujiwara, T. C. Schulthess, and W. H. Butler. Phys. Rev. B, 60:13237, 1999. 
This figure "fig1.jpg" is available in "jpg" format from: http://arxiv.org/ps/cond-mat/0510203v1 